\title{
Committee on Publication Ethics: the COPE Report 1999 Guidelines on good publication practice
}

Reference: Committee on Publication Ethics. The COPE Report 1999. Guidelines on good publication practice. London: BMJ Books, 1999:43-47. www.publicationethics.org

Why the guidelines were developed

COPE was founded in 1997 to address breaches of research and publication ethics. A voluntary body providing a discussion forum and advice for scientific editors, it aims to find practical ways of dealing with the issues, and to develop good practice.

We thought it essential to attempt to define best practice in the ethics of scientific publishing. These guidelines should be useful for authors, editors, editorial board members, readers, owners of journals, and publishers.

Intellectual honesty should be actively encouraged in all medical and scientific courses of study, and used to inform publication ethics and prevent misconduct. It is with that in mind that these guidelines have been produced.

Details of other guidelines on the ethics of research and published codes of conduct are listed in the Appendix.

How the guidelines were developed

The guidelines were developed from a preliminary version drafted by individual members of the committee, which was then submitted to extensive consultation. They address: study design and ethical approval, data analysis, authorship, conflict of interests, the peer review process, redundant publication, plagiarism, duties of editors, media relations, advertising, and how to deal with misconduct.

What they aim to do

These guidelines are intended to be advisory rather than prescriptive, and to evolve over time. We hope that they will be disseminated widely, endorsed by editors, and refined by those who use them.

(1) Study design and ethical approval

(2) Data analysis

(3) Authorship

(4) Conflicts of interest

(5) Peer review

(6) Redundant publication

(7) Plagiarism

(8) Duties of editors

(9) Media relations

(10) Advertising

Dealing with misconduct

Appendix

\section{Acknowledgement}

The following are gratefully acknowledged for their contribution to the drafting of these guidelines: Philip Fulford (Coordinator), Professor Michael Doherty, Ms Jane Smith, Dr Richard Smith, Dr Fiona Godlee, Dr Peter Wilmshurst, Dr Richard Horton, Professor Michael Farthing, Other members of COPE, Delegates to the meeting on April 27 1999, other corresponding editors.

\section{(1) Study design and ethical approval}

\section{Definition}

Good research should be well justified, well planned, appropriately designed, and ethically approved. To conduct research to a lower standard may constitute misconduct.
Action

(1) Laboratory and clinical research should be driven by protocol; pilot studies should have a written rationale.

(2) Research protocols should seek to answer specific questions, rather than just collect data.

(3) Protocols must be carefully agreed by all contributors and collaborators, including, if appropriate, the participants.

(4) The final protocol should form part of the research record.

(5) Early agreement on the precise roles of the contributors and collaborators, and on matters of authorship and publication, is advised.

(6) Statistical issues should be considered early in study design, including power calculations, to ensure there are neither too few nor too many participants.

(7) Formal and documented ethical approval from an appropriately constituted research ethics committee is required for all studies involving people, medical records, and anonymised human tissues.

(8) Use of human tissues in research should conform to the highest ethical standards, such as those recommended by the Nuffield Council on Bioethics.

(9) Fully informed consent should always be sought. It may not always be possible, however, and in such circumstances, an appropriately constituted research ethics committee should decide if this is ethically acceptable.

(10) When participants are unable to give fully informed consent, research should follow international guidelines, such as those of the Council for International Organisations of Medical Sciences (CIOMS).

(11) Animal experiments require full compliance with local, national, ethical, and regulatory principles, and local licensing arrangements. International standards vary.

(12) Formal supervision, usually the responsibility of the principal investigator, should be provided for all research projects: this must include quality control, and the frequent review and long term retention (may be up to 15 years) of all records and primary outputs.

\section{(2) Data analysis}

Definition

Data should be appropriately analysed, but inappropriate analysis does not necessarily amount to misconduct. Fabrication and falsification of data do constitute misconduct.

\section{Action}

(1) All sources and methods used to obtain and analyse data, including any electronic pre-processing, should be fully disclosed; detailed explanations should be provided for any exclusions.

(2) Methods of analysis must be explained in detail, and referenced, if they are not in common use.

(3) The post hoc analysis of subgroups is acceptable, as long as this is disclosed. Failure to disclose that the analysis was post hoc is unacceptable.

(4) The discussion section of a paper should mention any issues of bias which have been considered, and explain how they have been dealt with in the design and interpretation of the study. 


\section{(3) Authorship}

\section{Definition}

There is no universally agreed definition of authorship, although attempts have been made (see Appendix). As a minimum, authors should take responsibility for a particular section of the study.

\section{Action}

(1) The award of authorship should balance intellectual contributions to the conception, design, analysis and writing of the study against the collection of data and other routine work. If there is no task that can reasonably be attributed to a particular individual, then that individual should not be credited with authorship.

(2) To avoid disputes over attribution of academic credit, it is helpful to decide early on in the planning of a research project who will be credited as authors, as contributors, and who will be acknowledged.

(3) All authors must take public responsibility for the content of their paper. The multidisciplinary nature of much research can make this difficult, but this can be resolved by the disclosure of individual contributions.

(4) Careful reading of the target journal's "Advice to authors" is advised, in the light of current uncertainties.

\section{(4) Conflicts of interest}

\section{Definition}

Conflicts of interest comprise those which may not be fully apparent and which may influence the judgment of author, reviewers, and editors.

They have been described as those which, when revealed later, would make a reasonable reader feel misled or deceived.

They may be personal, commercial, political, academic or financial.

"Financial" interests may include employment, research funding, stock or share ownership, payment for lectures or travel, consultancies and company support for staff.

\section{Action}

(1) Such interests, where relevant, must be declared to editors by researchers, authors, and reviewers.

(2) Editors should also disclose relevant conflicts of interest to their readers. If in doubt, disclose. Sometimes editors may need to withdraw from the review and selection process for the relevant submission.

\section{(5) Peer review}

\section{Definition}

Peer reviewers are external experts chosen by editors to provide written opinions, with the aim of improving the study.

Working methods vary from journal to journal, but some use open procedures in which the name of the reviewer is disclosed, together with the full or "edited" report.

\section{Action}

(1) Suggestions from authors as to who might act as reviewers are often useful, but there should be no obligation on editors to use those suggested.

(2) The duty of confidentiality in the assessment of a manuscript must be maintained by expert reviewers, and this extends to reviewers' colleagues who may be asked (with the editor's permission) to give opinions on specific sections.

(3) The submitted manuscript should not be retained or copied.

(4) Reviewers and editors should not make any use of the data, arguments, or interpretations, unless they have the authors' permission.
(5) Reviewers should provide speedy, accurate, courteous, unbiased, and justifiable reports.

(6) If reviewers suspect misconduct, they should write in confidence to the editor.

(7) Journals should publish accurate descriptions of their peer review, selection, and appeals processes.

(8) Journals should also provide regular audits of their acceptance rates and publication times.

\section{(6) Redundant publication}

\section{Definition}

Redundant publication occurs when two or more papers, without full cross reference, share the same hypothesis, data, discussion points, or conclusions.

Action

(1) Published studies do not need to be repeated unless further confirmation is required.

(2) Previous publication of an abstract during the proceedings of meetings does not preclude subsequent submission for publication, but full disclosure should be made at the time of submission.

(3) Re-publication of a paper in another language is acceptable, provided that there is full and prominent disclosure of its original source at the time of submission. (4) At the time of submission, authors should disclose details of related papers, even if in a different language, and similar papers in press.

\section{(7) Plagiarism}

\section{Definition}

Plagiarism ranges from the unreferenced use of others' published and unpublished ideas, including research grant applications to submission under "new" authorship of a complete paper, sometimes in a different language.

It may occur at any stage of planning, research, writing, or publication: it applies to print and electronic versions.

Action

(1) All sources should be disclosed, and if large amounts of other people's written or illustrative material is to be used, permission must be sought.

\section{(8) Duties of editors}

\section{Definition}

Editors are the stewards of journals. They usually take over their journal from the previous editor(s) and always want to hand over the journal in good shape.

Most editors provide direction for the journal and build a strong management team. They must consider and balance the interests of many constituents, including readers, authors, staff, owners, editorial board members, advertisers and the media.

\section{Action}

(1) Editors' decisions to accept or reject a paper for publication should be based only on the paper's importance, originality, and clarity, and the study's relevance to the remit of the journal.

(2) Studies that challenge previous work published in the journal should be given an especially sympathetic hearing.

(3) Studies reporting negative results should not be excluded.

(4) All original studies should be peer reviewed before publication, taking into full account possible bias due to related or conflicting interests.

(5) Editors must treat all submitted papers as confidential. (6) When a published paper is subsequently found to contain major flaws, editors must accept responsibility for correcting the record prominently and promptly. 


\section{(9) Media relations}

Definition

Medical research findings are of increasing interest to the print and broadcast media.

Journalists may attend scientific meetings at which preliminary research findings are presented, leading to their premature publication in the mass media.

Action

(1) Authors approached by the media should give as balanced an account of their work as possible, ensuring that they point out where evidence ends and speculation begins.

(2) Simultaneous publication in the mass media and a peer reviewed journal is advised, as this usually means that enough evidence and data have been provided to satisfy informed and critical readers.

(3) Where this is not possible, authors should help journalists to produce accurate reports, but refrain from supplying additional data.

(4) All efforts should be made to ensure that patients who have helped with the research should be informed of the results by the authors before the mass media, especially if there are clinical implications.

(5) Authors should be advised by the organisers if journalists are to attend scientific meetings.

(6) It may be helpful to authors to be advised of any media policies operated by the journal in which their work is to be published.

\section{(10) Advertising}

Definition

Many scientific journals and meetings derive significant income from advertising.

Reprints may also be lucrative.

Action

(1) Editorial decisions must not be influenced by advertising revenue or reprint potential: editorial and advertising administration must be clearly separated.

(2) Advertisements that mislead must be refused, and editors must be willing to publish criticisms, according to the same criteria used for material in the rest of the journal.

(3) Reprints should be published as they appear in the journal unless a correction is to be added.

\section{Dealing with misconduct}

\section{(1) Principles}

(1) The general principle confirming misconduct is intention to cause others to regard as true that which is not true.

(2) The examination of misconduct must therefore focus, not only on the particular act or omission, but also on the intention of the researcher, author, editor, reviewer or publisher involved.

(3) Deception may be by intention, by reckless disregard of possible consequences, or by negligence. It is implicit, therefore, that "best practice" requires complete honesty, with full disclosure.

(4) Codes of practice may raise awareness, but can never be exhaustive.

(2) Investigating misconduct

(1) Editors should not simply reject papers that raise questions of misconduct. They are ethically obliged to pursue the case. However, knowing how to investigate and respond to possible cases of misconduct is difficult.

(2) COPE is always willing to advise, but for legal reasons, can only advise on anonymised cases.

(3) It is for the editor to decide what action to take.
(3) Serious misconduct

(1) Editors must take all allegations and suspicions of misconduct seriously, but they must recognise that they do not usually have either the legal legitimacy or the means to conduct investigations into serious cases.

(2) The editor must decide when to alert the employers of the accused author(s).

(3) Some evidence is required, but if employers have a process for investigating accusations - as they are increasingly required to do-then editors do not need to assemble a complete case. Indeed, it may be ethically unsound for editors to do so, because such action usually means consulting experts, so spreading abroad serious questions about the author(s).

(4) If editors are presented with convincing evidenceperhaps by reviewers - of serious misconduct, they should immediately pass this on to the employers, notifying the author(s) that they are doing so.

(5) If accusations of serious misconduct are not accompanied by convincing evidence, then editors should confidentially seek expert advice.

(6) If the experts raise serious questions about the research, then editors should notify the employers.

(7) If the experts find no evidence of misconduct, the editorial processes should proceed in the normal way.

(8) If presented with convincing evidence of serious misconduct, where there is no employer to whom this can be referred, and the author(s) are registered doctors, cases can be referred to the General Medical Council.

(9) If, however, there is no organisation with the legitimacy and the means to conduct an investigation, then the editor may decide that the case is sufficiently important to warrant publishing something in the journal. Legal advice will then be essential.

(10) If editors are convinced that an employer has not conducted an adequate investigation of a serious accusation, they may feel that publication of a notice in the journal is warranted. Legal advice will be essential.

(11) Authors should be given the opportunity to respond to accusations of serious misconduct.

(4) Less serious misconduct

(1) Editors may judge that it is not necessary to involve employers in less serious cases of misconduct, such as redundant publication, deception over authorship, or failure to declare conflict of interest. Sometimes the evidence may speak for itself, although it may be wise to appoint an independent expert.

(2) Editors should remember that accusations of even minor misconduct may have serious implications for the author(s), and it may then be necessary to ask the employers to investigate.

(3) Authors should be given the opportunity to respond to any charge of minor misconduct.

(4) If convinced of wrongdoing, editors may wish to adopt some of the sanctions outlined below.

\section{(5) Sanctions}

Sanctions may be applied separately or combined. The following are ranked in approximate order of severity:

(1) A letter of explanation (and education) to the authors, where there appears to be a genuine misunderstanding of principles.

(2) A letter of reprimand and warning as to future conduct.

(3) A formal letter to the relevant head of institution or funding body.

(4) Publication of a notice of redundant publication or plagiarism.

(5) An editorial giving full details of the misconduct.

(6) Refusal to accept future submissions from the 
individual, unit, or institution responsible for the misconduct, for a stated period.

(7) Formal withdrawal or retraction of the paper from the scientific literature, informing other editors and the indexing authorities.

(8) Reporting the case to the General Medical Council, or other such authority or organisation which can investigate and act with due process.

\section{Appendix}

The Association of the British Pharmaceutical Industry. Facilities for non patient volunteer studies. London: APBI, 1989.

The Association of the British Pharmaceutical Industry. Guidelines for medical experiments in non patient human volunteers. London: ABPI, 1990.

ABPI fact sheets and guidance notes:

Clinical trials and compensation guidelines, January 1991.

Guidelines for phase IV clinical trials, September 1993.

Guidelines on the conduct of investigator site audits, January 1994.

Relationship between the medical profession and the pharmaceutical industry, June 1994.

Good clinical trial practice, November 1995.

Patient information and consents for clinical trials, May 1997.

Guidelines on the structure of a formal agreement to conduct sponsored clinical research, July 1998.

Good clinical research practice, July 1998.

Council for International Organisations of Medical Sciences (CIOMS). International Guidelines for Ethical Review of Epidemiological Studies. Geneva: WHO, 1991.
General Medical Council. Good medical practice guidelines series:

Consent, February 1999.

Confidentiality, October 1995.

Transplantation of organs from live donors, November 1992.

International Committee of Medical Journal Editors (ICMJE). Uniform requirements for manuscripts submitted to biomedical journals. $\mathscr{f} A M A$ 1997;277:927-34.

Medical Research Council. Policy and procedure for inquiring into allegations of scientific misconduct. London: MRC, 1997.

Medical Research Council. The ethical conduct of research on the mentally incapacitated. London: MRC, 1991.

Medical Research Council. The ethical conduct of research on children. London: MRC, 1991.

Medical Research Council. Responsibility in the use of animals in medical research. London: MRC, 1993.

Medical Research Council. Responsibility in the use of personal medical information for research. Principles and guidelines to practice. London: MRC, 1985.

Medical Research Council. MRC Guidelines for good clinical practice in clinical trials. London: MRC, 1998.

Medical Research Council. Principles in the assessment and conduct of medical research and publicising results. London: MRC, 1995.

Nuffield Council on Bioethics. Human tissue: ethical and legal issues. London: Nuffield Council on Bioethics, 1995.

Royal College of Physicians. Research involving patients. London: RCP, 1990. 